OnLine Journal of Biological Sciences 10 (1): 1-10, 2010

ISSN 1608-4217

(C) 2010 Science Publications

\title{
Bheri-A Unique Example of Biological Complex System
}

\author{
${ }^{1}$ Indranil Mukherjee, ${ }^{2}$ Pranami Bhaumik, ${ }^{2}$ Madhusmita Mishra, \\ ${ }^{3}$ Ashoke Ranjan Thakur and ${ }^{2}$ Shaon Ray Chaudhuri \\ ${ }^{1}$ School of Management and Sciences, \\ ${ }^{2}$ Department of Biotechnology, \\ West Bengal University of Technology, BF-142, Sector-1, Salt Lake, Calcutta-700064, India \\ ${ }^{3}$ Vice Chancellor's Office, West Bengal State University (Barasat, North 24 Parganas), Berunanpukuria, \\ P.O. Malikapur, North 24 Parganas, Calcutta-700126, India
}

\begin{abstract}
Problem statement: This study is a unique attempt to characterize Bheri (shallow flat bottom waste water fed fishery) as a complex biological system. Approach: The characterization was done both at the qualitative and the quantitative levels. The diverse microbial community and the associated emergent behaviors resulting in integrated resource recovery by the Bheris were analyzed under the lens of complexity to gain an understanding about the qualitative features. To carry out quantitative characterization, ideas of scaling and power law relationships were emphasized through statistical analysis of observed data. Results: On comparing the plankton diversity of the Bheris with rain water and waste water fed ponds located within Calcutta (India) as well as Dhaka (Bangladesh), the former indicated clear evidence of exhibiting power law behavior (which is a strong signature of complexity). The adequacy of observations was reflected in the saturation of the curves obtained on plotting the number of observations against the Operational Taxonomic Unit (OTU). Conclusion: This study clearly reveals Bheri as a biological complex system both at qualitative and quantitative levels as compared to rain water as well as waste water fed ponds.
\end{abstract}

Key words:Bheri, power law, biological complex system, Operational Taxonomic Unit (OTU), Integrated Resource Recovery (IRR), bioremediation

\section{INTRODUCTION}

Complexity is a relatively new concept. The notion of a complex system is not yet precisely delineated (Yaneer, 1997; GellMann, 1995). It is probably easier to convey the meaning of complexity by describing certain typical properties of complex system. The search for the existence of these properties in any system can become the determining factor in characterizing such a system as complex. The analysis for complexity can be done either at the qualitative level taking into consideration one or more of these properties or at the quantitative level using power laws/scaling relationships that are characteristic features of complex system occurring in diverse areas of natural and social sciences.

Although a precise definition of complexity, involving necessary and sufficient conditions are somewhat difficult to formulate, some of the generic features of complexity are as follows:
A complex system (unit) must contain many constituents (subunits). These subunits must be interdependent (at least some of the time). The interactions between the subunits must be nonlinear (at least some of the time).

A complex system possesses a structure spanning several scales.

The properties of a complex system, observed at a particular scale, are said to be emergent in the sense that such properties cannot be explained when we study individually every constituent of this scale, each of which may also be a complex system made-up of finer scales. It is a new phenomenon special to the scale considered and arises from the global interaction between the scale's constituents.

The combination of structure and emergence leads to self organization, which is the outcome when an emerging behavior has the effect of changing the structure or creating a new structure. Each higher level has its own scale and each new level has new kinds of relationships and properties. A complex system at one

Corresponding Author: Shaon Ray Chaudhuri, Department of Biotechnology, West Bengal University of Technology, BF-142, Sector-1, Salt Lake, Calcutta-700064, India Tel: 00919831034236 Fax: 00913323341030 
level is made up of lower level complex systems interacting and creating the higher level order. Selforganization is not a strictly nested phenomenon; complex web of interactions may exist at all levels. (Yaneer, 1997; Gell-Mann, 1995; Baranger, 2009).

There is no single point of control in a complex system. Complex systems show global coordinated behavior, without the presence of any distinct global controller.

Generally some unique characteristics of an ecological system are responsible for its complexity. Some of these characteristics are large number of diverse components, nonlinear interactions, scale multiplicity, spatial heterogeneity, emergent properties, unexpected dynamics and self-organization. The complexity often takes the form of modularity in structure and functionality. Both hierarchical theory and empirical evidence confirm this (Wu and David, 2002), e.g., there are indeed a wide range of physical, biological and man-made components that interact in building up an ecosystem.

There are different statistical measures to describe the complexity of an ecological system. This can be done by quantifying the degree of diversity and its dependence on space or time, the organization of the biological interactions or the response of the system to perturbations (Sole et al., 2001a; 2001b).

A simple power-law or scaling relationship can often describe the complexity aspect inherent in biological systems, viz.:

$$
\mathrm{Y}=\mathrm{Y}_{0} \mathrm{x}^{\mathrm{a}}
$$

Where:

$\mathrm{Y}=$ Some response or dependent variable

$\mathrm{x}=$ An independent or explanatory variable

$\mathrm{Y}_{0}=\mathrm{A}$ normalization constant

$\mathrm{a}=$ The scaling exponent

At higher levels of organizations such as at the level of populations, communities and ecosystems, such scaling relationships are fairly common (Marquet $e t$ al., 2005).

Studies have been carried out to understand the microbial as well as eukaryotic diversity in terms of taxa-area relationships from the point of view of power law and spatial scaling (Brown et al., 2002; Finlay, 2002; Green et al., 2004; Horner et al., 2004). Despite an increasing awareness that spatial patterning of soil microbiota can have important aboveground consequences in regards to plant community structure and ecosystem functioning (Green et al., 2004; Balser et al., 2001; Callaway et al., 2004), microbial diversity patterns are largely unknown. Inadequate sampling has been a major limitation and it is only recently that researchers are beginning to explore emergent patterns and principles that may be common to microbes, plants and animals. Concepts of scaling have been widely used in other biological systems as well (Makarieva et al., 2005a). Extensive studies have been carried out regarding metabolic scaling laws focused on the model of West, Brown and Enquist (WBE), limitations have been identified and possible modifications discussed (Makarieva et al., 2005b; 2005c). In another related study, (Chen et al., 2005) have discussed how monitoring multiple species at multiple spatial and temporal scales is necessary to understand multi-species interactions and community dynamics.

Power laws are characteristic of fractal objects because these are the only functions displaying invariance under scale change. Statistical properties of ecological and geologic fractals as well as fractal geometry applications in description and analysis of patch patterns and patch dynamics have been investigated extensively ( $\mathrm{Li}, 2000$; Loehle and $\mathrm{Li}$, 1996).

Two theoretically and empirically important characteristics are associated with power-law relationships; firstly, power-laws and scaling relationships are scale-invariant, that is they posses the same statistical properties at any scale. This property makes scaling and power-law relationships very well suited for the study of ecological systems, which show variability at different temporal, spatial and organizational scales such that there is no single 'correct scale' for their analysis. The second important feature is the notion of universality. To understand this feature, it is useful to examine the biomass size distribution of pelagic communities, which have already been studied by many groups of researchers. The most common representation of biomass size spectra is in a form where every individual in the system is assigned to one of a series of size classes represented on a logarithmic scale conforming to an unnormalized spectrum. Evidence to date shows that oligotrophic ecosystems close to a steady state present more or less linear normalized biomass size spectra (log-log scale). Thus, scaling and power-laws point to the action of universal or law-like phenomena allowing the study of ecological systems even in the absence of detailed knowledge on demography and dynamics. These tools provide a fresh perspective to tackle ecological complexity, from individuals to ecosystems (Marquet et al., 2005). Application of power-law and scaling relationship are evident in many scientific works such as to determine the abundance-range size 
relationship, abundance-body size relationship, range size-latitude relationship, body size-latitude relationship, species-area relationship, local-regional species richness relationship, species-energy relationship, latitudinal gradient in species richness (David and Kevin, 2004).

Waste water fed fish ponds are a common feature in different parts of the world. The practice initially started in Germany and was soon imbibed by the Chinese followed by other countries. These water bodies in West Bengal, India (called Bheris) have a distinctly different architecture resulting in extensive purification of waste along with integrated resource recovery (Ray Chaudhuri et al., 2008a). Bheri, the shallow (50-150 $\mathrm{cm}$ ), flat bottom, waste water fed fish pond, is a unique ecosystem. The traditional knowledge, on detailed analysis, revealed interesting scientific basis behind this age old practice. The shallow basin allows full vertical circulation of water to the surface where algal blooms can occur. This depth provides a better ratio between pond volume and pond surface as compared to deeper pond, thus making this architecture favorable for photosynthesis (Pradhan et al., 2008). The alkalinity due to lime addition during pond preparation results in pathogen/fecal coliform content reduction by $96-99 \%$ (Pradhan et al., 2008). Abundant quantity of algal photosynthetic oxygen generated in the pond is one of the reasons for the purification of sewage (Barabasi and Stanley, 1995). Bheri causes detoxification of waste water by reducing heavy metals up to $25-99 \%$, while maintaining the biological oxygen demand at above $80 \%$. Being shallow, Bheri allows solar radiation to penetrate to the bottom and thereby facilitates photosynthesis. The high alkalinity stimulates the production of phytoplanktons, a primary product in the fish food chain. The essential factors in the purification process are the shallow depth allowing penetration of uniform sun rays to the bottom of the pond; the wind causing oxygenation through stirring of the water; growth of multiple forms of plankton, algae and bacteria resulting in active bioremediation as well as abundance of water hyacinth leading to metal accumulation along the different plant parts (Ray Chaudhuri et al., 2008c).

A significant contribution to the purification process is made by the planktonic species. The solar energy is trapped by a dense population of planktons which then get consumed by the fishes. The planktons play an important role in degrading the organic matter. Different planktons are known to play specific roles in bioremedial processes like metal accumulation, degradation of several aromatic hydrocarbons, herbicides and insecticides. A comparative study on the diversity, useful varieties and total number of planktons in rain water ponds from West Bengal, waste water fed fish ponds (called Dighi and Khamar) from Bangladesh (due to similar climatic conditions) as well as Bheris from West Bengal, India clearly indicated a richer diversity in case of the latter. The abundance of different planktons in Bheri, as compared to the Dighis, Khamars and fresh water ponds is in correlation with their active participation in the bioremediation process taking place in this complex ecosystem (Pradhan et al., 2008). However, overgrowth of planktons often becomes a problem for pond management since they cause algal bloom. At the critical phase of transition from plankton growth to plankton bloom, fish plays an important role by grazing on the plankton. The two fold role played by fishes is indeed crucial-they maintain a proper balance of the plankton population in the Bheri and also convert the available nutrients in the wastewater into readily consumable form that is fish (Ghosh, 1999).

There have been quite a few studies on the accumulation of metals in fishes generated through these means (Chatterjee et al., 2006; Ray Chaudhuri et $a l ., 2008 \mathrm{~b}$ ). The water and effluent generated from the Bheri are used for cultivation of vegetables, which, on examination using Energy Dispersive $X$ Ray Fluorescence, show no harmful metal accumulation (Ray Chaudhuri et al., 2007). A detailed analysis of the plankton varieties, plankton count, total coliform count, dissolved oxygen, conductivity and so on present in water of Bheris as compared to rain water fed ponds, Dighi as well as Khamars, emphasized the architecture of Bheri as a facilitator for waste water purification (Barabasi and Stanley, 1995). This explains the fact that though waste water fed fish ponds are a common feature in different parts of the world, yet not all work as efficiently as those at ECW (Ray Chaudhuri et al., 2008a). Studies have also revealed rich microbial diversity (Ray Chaudhuri and Thakur, 2006) in Bheris. Thus, Bheri being a complicated ecosystem from the functional and biodiversity aspects, this study intended to analyze it as a model of a biological complex system as compared to rain water ponds and Khamars.

\section{MATERIALS AND METHODS}

The quantitative characterization: In this study, attempts have been made to characterize Bheri as a complex system by utilizing the concepts of scaling and power law relationships. For this purpose, samples were collected from five different Bheris, five different rain water ponds (both from West Bengal India) and three different Khamars from Dhaka, Bangladesh. The data were analyzed in terms of the saturation curves 
obtained and the "straight lines" fitted by the least square method.

Data collection and analysis:

Collection of samples from the Bheris of East Calcutta Wetland: Water samples were collected from five different Bheris viz., Captain Bheri, Natar Bheri, Nuner Bheri (No. 1), Nuner Bheri (No. 2) and Charakdanga Bheri. The distances between Charakdanga Bheri and the others, viz., Captain Bheri, Natar Bheri, Nuner Bheri 1, Nuner Bheri 2 are 1771, 2142, 1514 and 1457 meters respectively. Thirty aliquots of water samples were collected from different parts of each Bheri. The samples were collected in sterile falcon tubes of $50 \mathrm{~mL}$ each from a depth of 1.5 $2.0 \mathrm{~cm}$ below the surface of the water.

Collection of samples from Rainwater ponds and Khamar: The collection procedure was same as that for Bheris. The difference was mainly in the selection of the spots which were more dispersed. The samples were carried to the laboratory at room temperature and maintained similarly until the analysis was completed.

Saturation study: Water in each falcon was mixed by inversion and $20 \mu \mathrm{L}$ was observed under the light microscope. The number of observations and the different varieties of microscopic bodies observed were noted and a graph was plotted with number of observations on the $\mathrm{X}$ axis and the total number of newer varieties observed with each observation on the $\mathrm{Y}$ axis. The resultant curve indicates whether saturation in screening the diversity has been obtained. This process was repeated for each Bheri, rainwater pond and Khamar.

For determining the plankton diversity in the Bheris the following procedure was adopted: the water $(100 \mu \mathrm{L})$ from different aliquots were pooled and harvested at $10,000 \mathrm{~g}$ for $10 \mathrm{~min}$. The supernatant was discarded and the pellet was resuspended in $100 \mu \mathrm{L}$ of distilled water and $20 \mu \mathrm{L}$ of it was used to visualize the suspension under light microscope.

Power law: For quantitative analysis of the system, power law technique was applied as done previously by other groups (Green et al., 2004; Green and Bohannan, 2006). The taxa-observation relationship is well approximated by a power law of the following form:

$$
\mathrm{OTU}_{\mathrm{a}}=\mathrm{OTU}_{\mathrm{A}}(\mathrm{a} / \mathrm{A})^{\mathrm{z}}
$$

Where:

$\mathrm{OTU}_{\mathrm{a}}=$ The number of species observed in the first observation
$\mathrm{OTU}_{\mathrm{A}}=$ The number of total species observed till that particular observation

a $=$ The first observation and so is equal to 1

A $=$ Any particular subsequent observation

Taking logarithm on both sides of Eq. 1 and rearranging, we get:

$\log \left(\mathrm{OTU}_{\mathrm{A}} / \mathrm{OTU}_{\mathrm{a}}\right)=\mathrm{z} \log (\mathrm{A} / \mathrm{a})$

Equation 2 gives a straight line in the variables $\log$ (A/a) and $\log \left(\mathrm{OTU}_{\mathrm{A}} / \mathrm{OTU}_{\mathrm{a}}\right)$, thereby permitting the use of linear regression. The detailed procedure for arriving at these values is described below: water samples were collected from the water bodies in 30 falcon tubes each. They were used $(20 \mu \mathrm{L})$ to observe the varieties of planktons in those water samples. The number of OTU was calculated. The value of $\log (\mathrm{A} / \mathrm{a})$ as well as $\log$ $\left(\mathrm{OTU}_{\mathrm{A}} / \mathrm{OTU}_{\mathrm{a}}\right)$ was calculated for each point of individual water bodies. The data was plotted with the former on the $\mathrm{X}$ and the latter on the $\mathrm{Y}$-axis.

Statistical analysis of data: Regression Analysis was employed to fit the "best" straight line to the observed data points with $\log (\mathrm{A} / \mathrm{a})$ and $\log \left(\mathrm{OTU}_{\mathrm{A}} / \mathrm{OTU}_{\mathrm{a}}\right)$ as the independent and dependent variables respectively. Values of the following quantities were obtained: slope and intercept of the "best" fit straight lines, coefficient of determination, standard errors for the slope and intercept.

The qualitative characterization: The bioremediation as well as integrated resource recovery occurring in the Bheris resulting in cleaner environment with better livelihood support was compared with the characteristic features of a complex system to unravel the association, if any.

\section{RESULTS}

Saturation curves: When the number of observations was plotted (X-axis) against the number of total new varieties, called Operational Taxonomic Unit (OTU) observed till a particular observation (Y-axis) for each water body, a saturation curve was obtained. A representative graph for the Bheris is shown in Fig. 1.

Alignment of data-points by least square fit: In the present research, the relationship between relative richness of species $\left(\mathrm{OTU}_{\mathrm{A}} / \mathrm{OTU}_{\mathrm{a}}\right)$ and relative observation (A/a) was scaled (Fig. 2). 


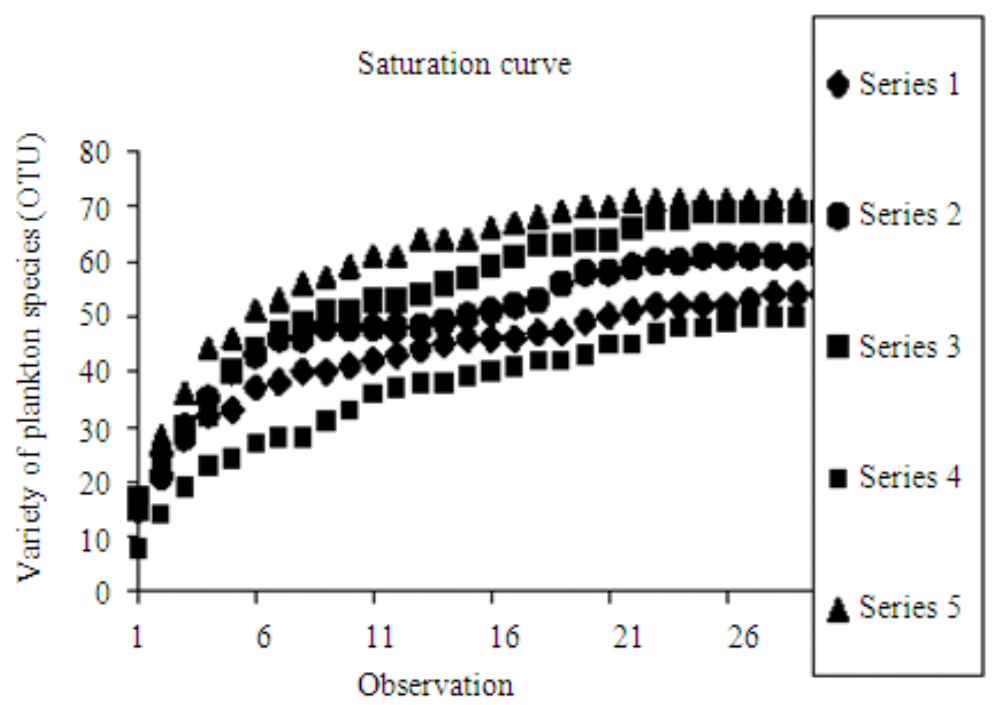

Fig. 1: The saturation curve for different Bheris. The number of plankton varieties visualized with each observation was plotted for all the five Bheris. Here each series stands for one Bheri. Series 1-5 represents Captain Bheri, Natar Bheri, Nuner Bheri (No. 1), Nuner Bheri (No. 2) and Charakdanga Bheri respectively
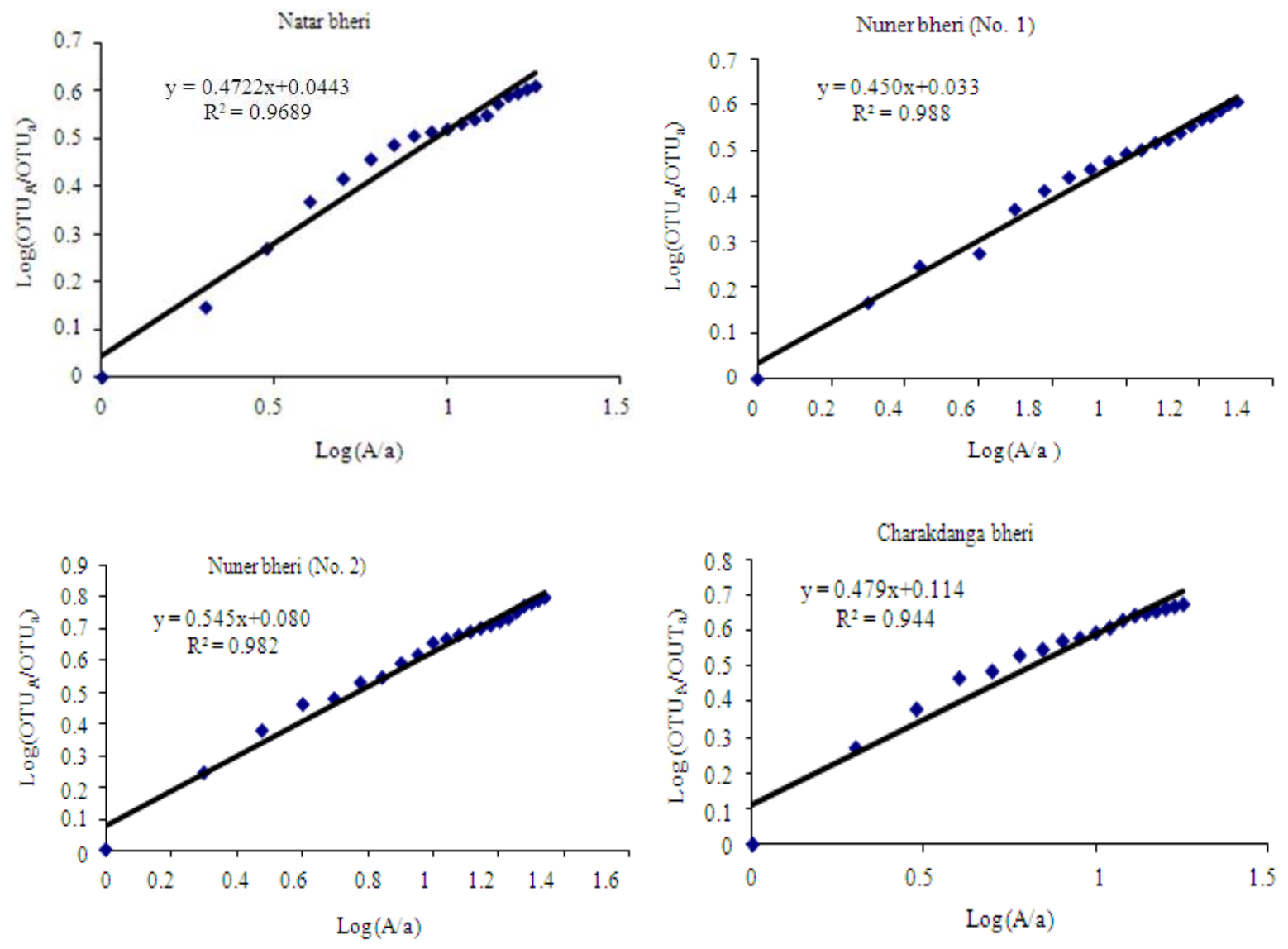
OnLine J. Biol. Sci., 10 (1): 1-10, 2010

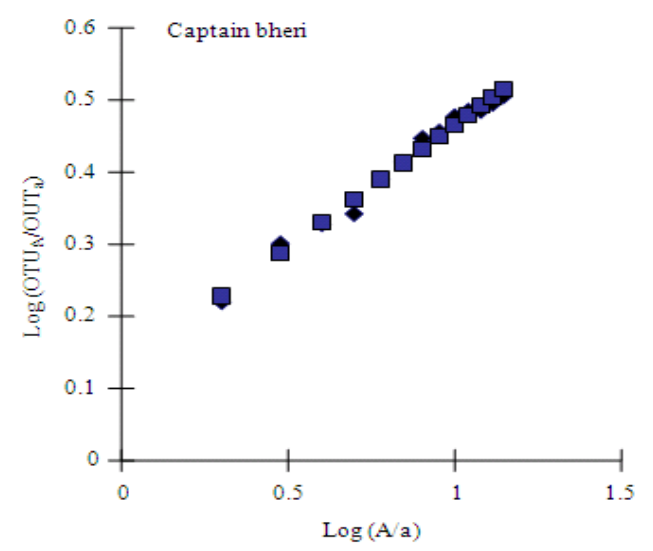

(A)
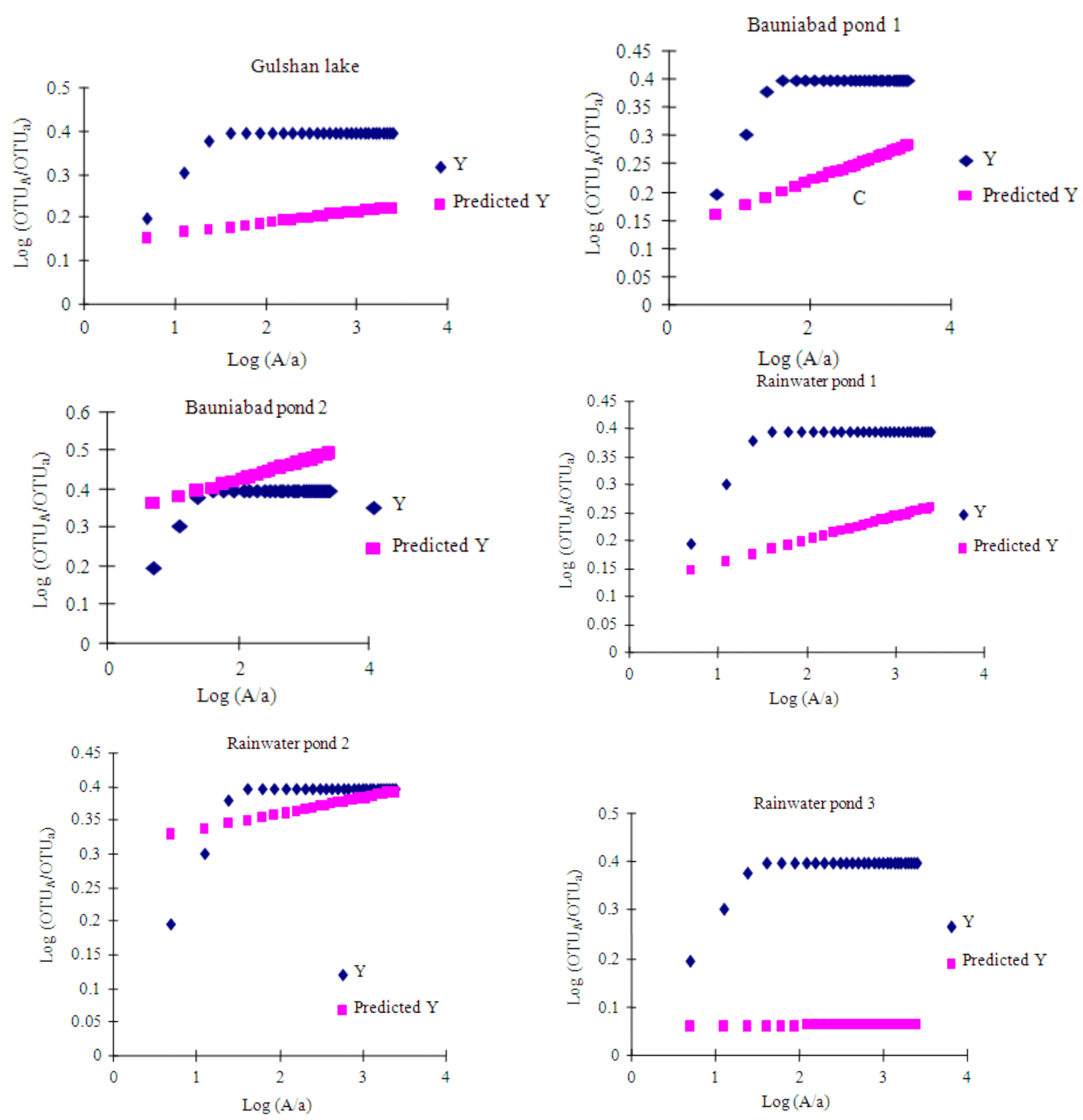
OnLine J. Biol. Sci., 10 (1): 1-10, 2010
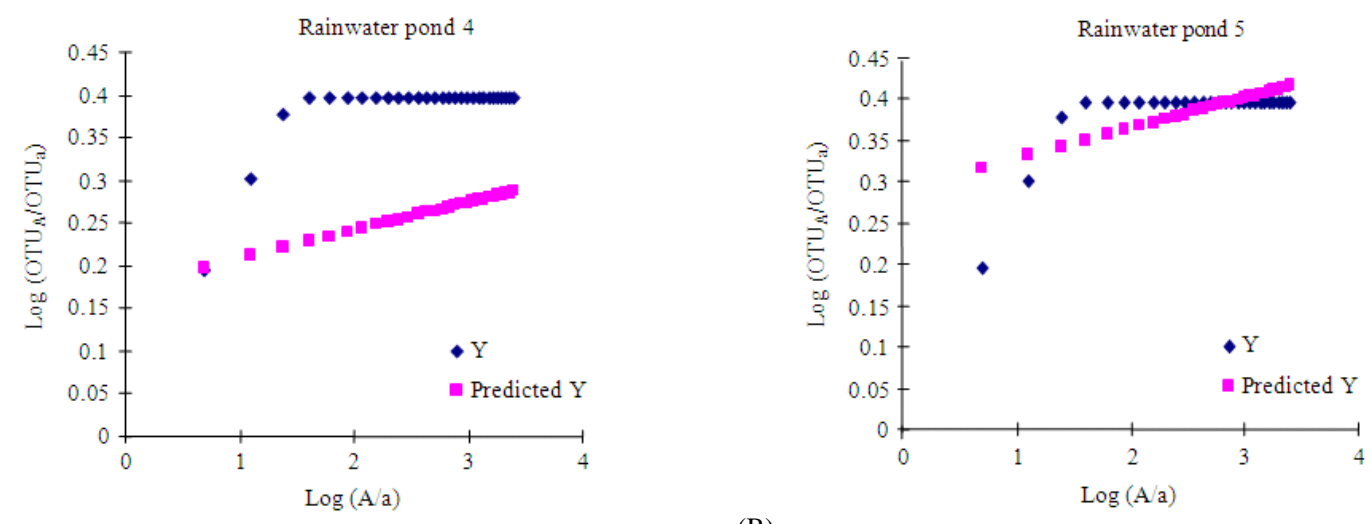

(B)

Fig. 2: Statistical analysis of observed data through least square estimation. (A) The best fitted straight line through the data points obtained from Natar Bheri, Nuner Bheri (No. 1), Nuner Bheri (No. 2), Charakdanga Bheri and Captain bheri by least square estimation indicates them to be non-linear complex system at the quantitative level. (B) Similar analysis for the other water bodies clearly indicate departure from straight line behavior pointing towards the inadequacy of power law description.

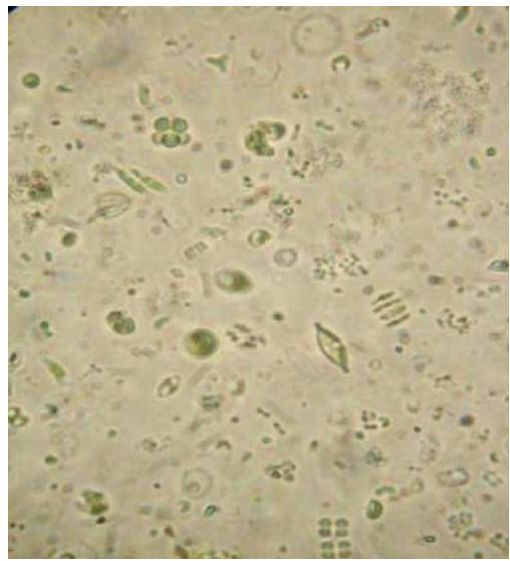

Fig. 3: Photograph of microscopic biota at 40X magnification. The species present in the image were identified as follows: Closterium sp., Scenedesmus sp., Tetraedron sp., Cosmarium sp., Microcystis sp., Cyclotella sp. Asterionella sp., Diatoma sp., Navicula sp., Nitzschia sp., Characiopsis sp., Chlamydomonas sp., Chlorella sp., Chlorococcum sp. Haematococcus sp., Kirchneriella sp., Micractinium sp., $\quad$ Merismopedia sp., Stephanodiscus sp

If power law is obeyed then the log-log plot should be a straight line. Linear regression was used to fit the "best" straight line using data on relative richness of species $\left(\mathrm{OTU}_{\mathrm{A}} / \mathrm{OTU}_{\mathrm{a}}\right)$ and relative observation $(\mathrm{A} / \mathrm{a})$. The slope $(\mathrm{m})$, intercept (c) and the coefficient of determination $\left(r^{2}\right)$ were calculated for each set (i.e., using data collected from each water body) in the form of a triplet of values as depicted below:

- Captain Bheri-(0.3400, 0.1250, 0.9860)

- Natar Bheri-(0.4392, 0.0775, 0.9550)

- $\quad$ Nuner Bheri (No. 1)-(0.4351, 0.0491, 0.9854)

- $\quad$ Nuner Bheri (No. 2)-(0.4984, 0.1315, 0.9911)

- Charakdanga Bheri-(0.3944, 0.1998, 0.9733)

- Gulshan lake-(0.0256, 0.1349, 0.5428)

- $\quad$ Bauniabad pond 1-(0.0449, 0.1272, 0.5348)

- Bauniabad pond 2-(0.0487, 0.3242, 0.6090)

- Rain water pond 1-(0.0416, 0.1169, 0.6831)

- Rain water pond 2-(0.0236, 0.3113, 0.3801)

- Rain water pond 3-(0.0027, 0.0542, 0.2516)

- Rain water pond 4-(0.0327, 0.1753, 0.5759)

- Rain water pond 5-(0.0370, 0.2897, 0.4314)

\section{DISCUSSION}

In Fig. 1 each series clearly depicted the adequacy of the screening. The data for Rain water ponds and Khamars were presented elsewhere (Pradhan et al., 2008). The saturation curve obviates the need for increasing the number of observations in each case.

The high values of the coefficient of determination in case of Bheris indicate that almost the entire variation in the dependent variable can be explained by assuming a linear functional form of the independent variable, which in turn validates the power law behavior (Fig. 2A). However the same for the other water bodies are much lower indicating that the log-log 
plot departs significantly from straight line behavior showing inadequacy of the power law behavior in case of these entities (Fig. 2B). Values of the slopes determined by this analysis agree with the result that the slope of the log-log graph in this setting should be within zero to one (Green et al., 2004). Results of other studies show the slope to be 0.134 for phytoplankton morphospecies in Aquatic Island (Smith et al., 2005).

Exhaustive statistical analyses were carried out for each data set; viz., regression statistics, residual output, standard errors, p-values and t-statistics for both slope and intercept of the "best" fitted straight line as well as a brief ANOVA with F statistics (data not shown).

This study started from the premise that the loglog plot should be a straight line in case of a complex system. Calculation of the values of $\mathrm{m}, \mathrm{c}$ and $\mathrm{r}^{2}$ clearly validate this hypothesis. From the above results (Fig. 2A and B) it is clear that inspite of being involved in fish production with microbial growth; Bheri, Khamar as well as rain water pond show distinct variation with only Bheri satisfying the power law behavior, a typical signature of complexity.

The qualitative characterization: It is also possible to characterize Bheri as a complex system by examining some features of Bheri at a qualitative level as the following points show:

- Bheri consists of multiple subunits in the form of bacteria, phytoplankton (Fig. 3), zooplankton, fish and water hyacinth. It reflects the property of a complex system which has multiple subunits

- The food chain described earlier explains the interaction between the subunits to ensure functioning of the system. It also indicates the structure (of the Bheri) which spans over several scales from microscopic bacteria to macroscopic fishes. The water hyacinth accumulates metal within its submerged as well as aerial parts, reducing the metal content of water. The water draining into the Bheri comes from domestic as well as industrial sectors causing heavy metal load in it. This metal load is reported to be purified through natural processes before it is finally released into river Hoogly (Smith et al., 2005). This can be correlated to the property of interaction being present among the different components of the Bheri having a structure spanning several scales

- The interaction between different subunits is essential for the completion of the processes operating within the Bheri. However, no single component behaves as the sole controller of the process. The treatment of the surface with lime is necessary to kill the pathogens while the other microbes grow removing metals and degrading wastes. The leveling of the bottom of the pond with the specific depth ensures uniform distribution of sunlight throughout the water body which is required for phytoplankton growth. The release of the fish fry at the optimal moment restricts plankton bloom and ensures that the latter is used as fish food. The smaller fishes are consumed by the larger ones which in turn are food for human beings. Thus each component is essential for the overall functioning of the system with none as the pivotal point. This is one of the defining features of a complex system

- The interaction of the individual components leads to resource recovery, waste management, employment/economic stability as well as revenue generation. Thus the interaction among the individual components leads to these emergent properties as depicted schematically (Fig. 4)

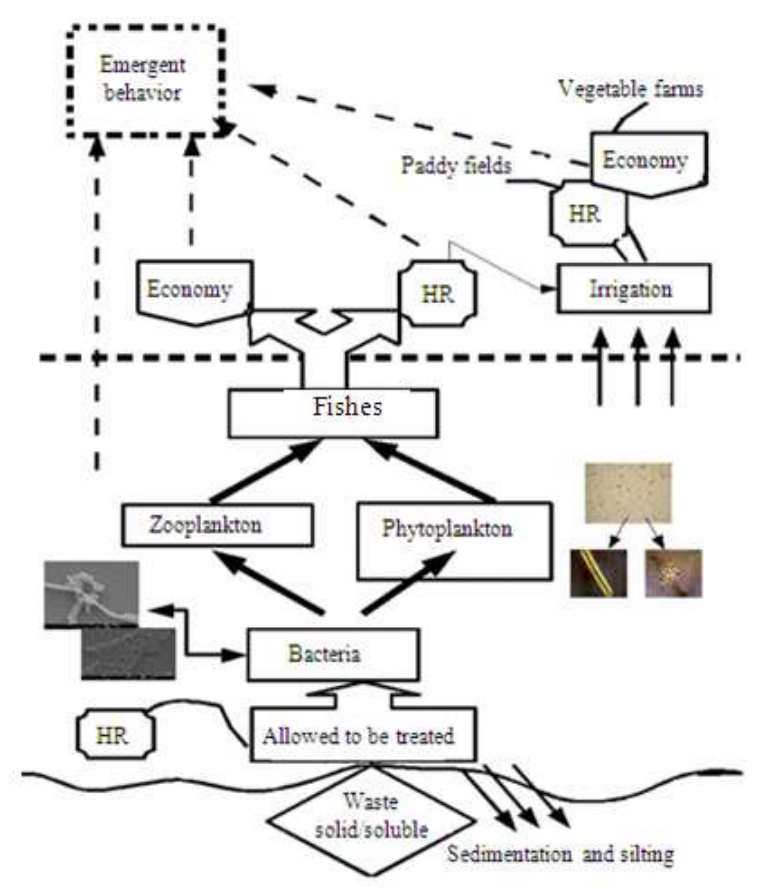

Fig. 4: Schematic representation of the various subunits of Bheri and their interaction resulting in the emergent behavior identifying Bheri as a biological complex system from the qualitative point of view. Here HR stands for human resource 
- The process operating at the Bheris leads to purification of soluble waste of the city. Here the waste gets converted to safe water for irrigation as well as fertile soil (the effluents from the bottom of the Bheri after each round of fish growth). This in turn provides employment to a large number of individuals (Ray Chaudhuri et al., 2009) and food (vegetables, paddy as well as fish) to an even greater population. Thus through its multifarious activities, Bheris self organize into integrated resource recovery units

Bheri consists of a large number of individual organisms belonging to thousands of species interacting with each other and with the abiotic environment both on spatial and temporal scales. These interactions are frequently non-linear in nature. The enormous physical and biological diversity, coupled with the non-linear interactions give rise to certain properties of the system that are emergent in nature. This study investigated Bheri as a complex system from the qualitative and the quantitative perspectives.

\section{CONCLUSION}

This study clearly reveals Bheri as a biological complex system both at qualitative and quantitative level as compared to rain water as well as waste water fed ponds.

\section{ACKNOWLEDGMENT}

The authors would like to acknowledge the financial support of Department of Atomic Energy, Government of India as well as West Bengal University of Technology. They would like to thank Bioinformatics Infrastructure Facility (DBT, GOI supported) of West Bengal University of Technology for its computational facility. They would also acknowledge Dr. Bilqis Amin Hoque, EPRC, Bangladesh for her help during sample collection.

\section{REFERENCES}

Balser, T.C., A.P. Kinzig and M.K. Firestone, 2001. Linking Soil Microbial Communities and Ecosystem Functioning. In: The Functional Consequences of Biodiversity, Kinzig, AP., S.W. Pacala and D. Tilman (Eds.). Princeton University Press, ISBN: 13: 978-0-691-08822-8, pp: 265-293.

Barabasi, A.L. and H.E. Stanley, 1995. Fractal Concepts in Surface Growth. Cambridge University Press, Cambridge, ISBN: 10: 0521483085, pp: 13-37.
Baranger, M., 2009. In chaos, complexity and entropy. http://necsi.org/projects/baranger/cce.pdf

Brown, J.H., V.K. Gupta, B.L. Li, B.T. Milne and C. Restrepo et al., 2002. The fractal nature of nature: Power laws, ecological complexity and biodiversity. Philos. Trans. R. Soc. Lond. B., 357: 619-626. DOI: 10.1098/rstb.2001.0993

Callaway, R.M., G.C. Thelen, A. Rodriguez and W.E. Holben, 2004. Soil biota and exotic plant invasion. Nature, 427: 731-733. DOI: 10.1038/nature02322

Chatterjee, S., B. Chattopadhyay and S.K. Mukhopadhyay, 2006. Trace metal distribution in tissues of cichlids (Oreochromis niloticus and O. mossambicus) collected from wastewater-fed fishponds in East Calcutta Wetlands, a Ramsar site. Acta Ichthyol. Piscat., 36: 119A-125A. http://www.aiep.pl/volumes/2000/6_2/pdf/05_738_ Chatterjee_et_al.pdf

Chen, X., B.L. Li and S.L. Collins, 2005. Multiscale monitoring of a multispecies case study: Two grass species at Sevilleta. Plant Ecol., 179: 149-154. DOI: $10.1007 / \mathrm{s} 11258-004-6802-\mathrm{Z}$

David, S., J.G. Kevin, 2004. Untangling ecological complexity on different scales of space and time. Basic Applied Ecol., 5: 389-400. DOI: 10.1016/j.baae.2004.08.001

Finlay, B.J., 2002. Global dispersal of free-living microbial eukaryote species. Science, 296: 10611063. DOI: $10.1126 /$ science. 1070710

Gell-Mann. M., 1995. What is complexity? Complexity, 1: 16-19. http://www.santafe.edu/ mgm/complexity.html

Ghosh, D., 1999. Participatory management in wastewater treatment and reuse in west Bengal. UWEP Occasional Paper. http://www.waste.nl/content/download/530/4112/fi le/publication\%20list.pdf

Green, J. and B.J.M. Bohannan, 2006. Microbial ecology spatial scaling of microbial biodiversity. Trends Ecol. Evol., 21: 501-507. DOI: 10.1016/j.tree.2006.06.012

Green, J.L., A.J. Holmes, M. Westoby, I. Oliver and D. Briscoe et al., 2004. Spatial scaling of microbial eukaryote diversity. Nature, 432: 747-750. DOI: 10.1038/nature03034

Horner, M.C., M. Lage, J.B. Hughes and J.M.B. Brendan, 2004. A taxa-area relationship for bacteria. Nature, 432: 750-753. DOI: 10.1038/nature03073

Li, B.L., 2000. Fractal geometry applications in description and analysis of patch patterns and patch dynamics. Ecol. Model., 132: 33-50. DOI: 10.1016/S0304-3800(00)00303-3 
Loehle, C. and B.L. Li., 1996. Statistical properties of ecological and geologic fractals. Ecol. Model., 85: 271-284. DOI: 10.1016/0304-3800(94)00177-4

Makarieva, A.M., V.G. Gorshkov and B.L. Li, 2005a. Why do population density and inverse home range scale differently with body size? Implications for ecosystem stability. Ecol. Complex., 2: 259-271. DOI: 10.1016/j.ecocom.2005.04.006

Makarieva, A.M., V.G. Gorshkov and B.L. Li, 2005 b. Revising the distributive networks models of West, Brown and Enquist (1997) and Banavar, Maritan and Rinaldo (1999): Metabolic inequity of living tissues provides clues for the observed allometric scaling rules. J. Theor. Biol., 237: 291-301. DOI: 10.1016/j.jtbi.2005.04.016

Makarieva, A.M., V.G. Gorshkov and B.L. Li, 2005 c. Biochemical universality of living matter and its metabolic implications. Funct. Ecol., 19: 547-557. DOI: $10.1111 / \mathrm{j} .1365-2435.2005 .01005 . \mathrm{x}$

Marquet, P.A., R.A. Quiñones, S. Abades, F. Labra and M. Tognelli et al., 2005. Scaling and powerlaws in ecological systems. J. Exp. Biol., 208: 1749-1769. DOI: 10.1242/jeb.01588

Pradhan, A., P. Bhaumik, S. Das, M. Mishra and S. Khanam et al., 2008. Phytoplankton diversity as indicator of water quality for fish cultivation. Am. J. Environ. Sci., 4: 406-411. http://www.scipub.org/fulltext/ajes/ajes44406411.pdf

Ray Chaudhuri, S. and A.R. Thakur, 2006. Microbial genetic resources mapping of east Calcutta wetlands. Curr. Sci., 91: 212-217. http://www.ias.ac.in/currsci/jul252006/212.pdf

Ray Chaudhuri, S., A.R. Thakur and C. Ulrischs, 2009. Studies on East Calcutta wetland: A Unique Biological Ecosystem. In Advances in Environmental Research, Albert T. Riley (Ed) Nova Science Publishers, Inc. Chapter 7, ISBN: 978-1-60876-168-5, Vol 3, pp: 1-17.

Ray Chaudhuri, S., M. Mishra, P. Nandy and A.R. Thakur, 2008a. Waste management: A case study of ongoing traditional practices at east Calcutta wetland. Am. J. Agric. Biosci., 3: 315-320. http://www.scipub.org/fulltext/AJAB/AJAB31315320.pdf
Ray Chaudhuri, S., M. Mishra, S. Salodkar, M. Sudarshan and A.R. Thakur, 2008b. Traditional aquaculture practice at East Calcutta Wetland: The safety assessment. Am. J. Environ. Sci., 4: 140-144. http://www.scipub.org/fulltext/ajes/ajes42173177.pdf

Ray Chaudhuri, S., S. Salodkar, M. Sudarshan, I. Mukherjee and A.R. Thakur, 2008c. Role of water hyacinth mediated phytoremediation in waste water purification at East Calcutta Wetland. Environ. Sci., 5: 53-62. DOI: 10.1080/15693430701833427

Ray Chaudhuri, S., S. Salodkar, M. Sudarsan and A.R. Thakur, 2007. Integrated resource recovery at East Calcutta Wetland: How safe ISB this? Am. J. Agric. $\quad$ Biosci., 2 : 75-80. http://www.scipub.org/fulltext/AJAB/AJAB227580.pdf

Smith, V.H., B.L. Foster, J.P. Grover, R.D. Holt and M.A. Leibold et al., 2005. Phytoplankton species richness scales consistently from laboratory microcosms to the world's oceans. PNAS, 102: 4393-4396. DOI: 10.1073/pnas.0500094102

Sole, R.V., D. Alonso and A. McKane, 2001a. Selforganized instability in complex ecosystems. Philos. Trans. R. Soc. Lond. B., 357: 667-681. DOI: 10.1098/rstb.2001.0992.

Sole, R.V., D. Alonso, J. Bascompte and S.C. Manrubia, 2001b. On the fractal nature of ecological and macroevolutionary dynamics. Fractals, 9: 1-16. DOI: $10.1142 / \mathrm{S} 0218348 X 01000592$.

$\mathrm{Wu}$, J. and J.L. David, 2002. A spatially explicit hierarchical approach to modeling complex ecological systems: Theory and applications. Ecol. Model., 153: 7-26. DOI: 10.1016/S03043800(01)00499-9

Yaneer, B.Y., 1997. Dynamics of complex systems Addison-Wesley, Reading, Mass. http://www.citeulike.org/article/3626540 accessed 17.04.09 